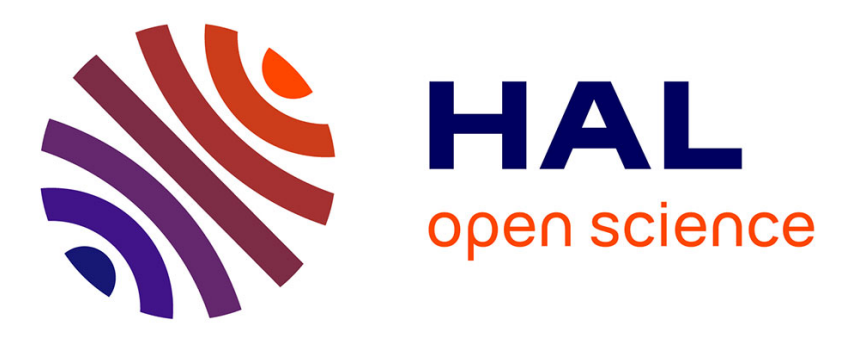

\title{
Three-dimensional optoacoustic tomography using a conventional ultrasound linear detector array: whole-body tomographic system for small animals.
}

Jérôme Gateau, Miguel Ángel Araque Caballero, Alexander Dima, Vasilis

Ntziachristos

\section{To cite this version:}

Jérôme Gateau, Miguel Ángel Araque Caballero, Alexander Dima, Vasilis Ntziachristos. Threedimensional optoacoustic tomography using a conventional ultrasound linear detector array: whole-body tomographic system for small animals.. Medical Physics, 2012, 40, pp.013302. 10.1118/1.4770292 . hal-00878254

\section{HAL Id: hal-00878254 https://hal.science/hal-00878254}

Submitted on 29 Jan 2014

HAL is a multi-disciplinary open access archive for the deposit and dissemination of scientific research documents, whether they are published or not. The documents may come from teaching and research institutions in France or abroad, or from public or private research centers.
L'archive ouverte pluridisciplinaire HAL, est destinée au dépôt et à la diffusion de documents scientifiques de niveau recherche, publiés ou non, émanant des établissements d'enseignement et de recherche français ou étrangers, des laboratoires publics ou privés. 


\title{
Three-dimensional opto-acoustic tomography using a conventional ultrasoundlinear detector array.
}

\author{
Whole-bodytomographic system for small animals
}

\author{
Authors: Jérôme Gateau ${ }^{1}$, Miguel Ángel Araque Caballero ${ }^{1}$, Alexander Dimaªnd
}

Vasilis Ntziachristos ${ }^{1}$.

${ }^{1}$ Institute for Biological and Medical Imaging (IBMI), Technical University of Munich and

Helmholtz Center Munich, Ingoldstädter Landstraße 1, 85764 Neuherberg, Germany

\begin{abstract}
Purpose: Optoacoustic imaging relies on the detection of ultrasonic waves induced by laser pulse excitations to map optical absorption in biological tissue. A tomographic geometry employing a conventional ultrasound linear detector array for volumetric optoacoustic imaging is reported. The geometry is based on a translate-rotate scanning motion of the detector array, and capitalizes on the geometrical characteristics of the transducer assembly to provide a large solid angular detection aperture. A system for three-dimensional whole-body optoacoustic tomography of small animals is implemented.

Methods: The detection geometry was tested using a 128-element linear array (5.0/7.0MHz, Acuson L7, Siemens), moved by steps with a rotation/translation stage assembly. Translationand rotation range of $13.5 \mathrm{~mm}$ and $180^{\circ}$ respectively were implemented. Optoacoustic emissions were induced in tissue-mimicking phantoms and ex-vivo mice using a pulsed laser operating in the near-IR spectral range at 760nm. Volumetric images were formed using a filtered back-projection algorithm.
\end{abstract}

Results: The resolution of the optoacoustic tomography system was measured to be better than $130 \mu$ min-plane and $330 \mu \mathrm{m}$ in elevation (full width half maximum), and to be homogenous along a $15 \mathrm{~mm}$ diameter cross-section due to the translate-rotate scanning geometry. Whole-body volumetric optoacoustic images of mice were performed exvivo, and imaged organs and blood vessels through the intact abdominal and head regions were correlated to the mouse anatomy.

Conclusions: Overall, the feasibility of three-dimensional and high-resolution whole-body optoacoustic imaging of small animal using a conventional linear array was demonstrated. Furthermore, the scanning geometry may be used for other linear arrays and is therefore expected to be of great interest for optoacoustic tomography at macroscopic 
29 and mesoscopic scale. Specifically, conventional detector arrays with higher central frequencies may be 30 investigated.

31

32 Keywords : optoacoustic, ultrasound, linear array,computed tomography, small animal imaging 


\section{I. INTRODUCTION}

Optoacoustic (photoacoustic) imagingis a noninvasive imaging modality for high-resolution

36 mapping of optical absorption in biological tissues ${ }^{1,2}$. The methodresolves optical absorption by detecting

37 broadband ultrasonic waves generated within the tissue following the absorption ofpulsed

38 illumination.The conversion from optical energy to mechanical wavesoccurs through the thermo-elastic

39 expansionof transiently heated absorbers, and its efficiency is strongly sensitive to the optical absorption

40 of the tissue structures. Similarly to optical imaging, the penetration of optoacoustic imaging, for

41 ultrasonic frequencies below $20-30 \mathrm{MHz}$, is primarily limited by the light attenuation in tissues ${ }^{3}$. However, optoacoustic imaging offers at mesoscopic and macroscopic scale a higher resolution than optical imaging, even when compared to pulsed illumination time-domain optical imaging ${ }^{4}$, thanks to the weak scattering of ultrasound wavesin soft tissues.Thereby, optoacoustic computed tomography (OAT) can provide optical contrast images with ultrasounddiffraction-limited resolution ${ }^{5}$. Additionally, light scatteringin biological tissue, and three-dimensional (3D) propagation of optoacoustic waves make macroscopicoptoacoustic imaging aninherently three-dimensional modality.

Volumetric images of the organ ${ }^{6-10}$ and vascular ${ }^{11-15}$ anatomies of healthy small animals, as well

49 as human breast ${ }^{13}$ and arm $^{16}$, have been obtained non-invasively usingthe endogenous optical absorption

50 contrast in tissue, and were found to provide 3D visualization of tissue and vascular architecture. 51 Localizationof molecular biomarkers ${ }^{10,17,18}$, induced thermal lesions ${ }^{19}$ or tumor vascularization ${ }^{20}$ have further shown great potential and application versatility of optoacoustic imaging.

Differentexperimentaltomographic configurationshave been proposed and implemented to obtain 3D macroscopic optoacoustic images ${ }^{21}$.In these configurations, ultrasonic detectors were arranged along spherical $^{6-9,}$, , cylindrical, circular ${ }^{5,22-28}$,or planar ${ }^{11,15-17,29,30}$ surfaces. The choice of the detection geometry is usually dictated experimentally by practicalconstrains such as the size of the imaged animal or tissue, and the positioning of the illuminationsystem. Overall, the geometricalarrangement of the 58 detectors influences the spatial resolution achieved in optoacoustic tomography and the visibility oftissue 59 structures, since limited view issues may arise from partial enclosure of the sample ${ }^{31,32}$. Besides the 60 detection geometry,detector properties such as spatial and electrical (bandwidth)responsesalso influence 61 the achieved resolution and image quality. For example, the spatial directivity of a detector,due to its 62 finite size and focusing properties, defines the angular aperture and the dimension of the volume covered 63 by its sensitivity field, and may lead to distortions of the collected optoacoustic signal ${ }^{5,33}$. Moreover, as 
optoacoutic waves exhibit a broadband spectrum, with peak frequencies corresponding to different object sizes ${ }^{34}$, the central frequency and bandwidth of the ultrasound detector defines the range of structure sizes that a given detector is able to resolve.

Many optoacoustic imaging implementationsrely on piezoelectric detectors and benefit from technologies developed for ultrasonic measurements. Arrays of detectors allow parallel acquisition and therefore reduce the acquisition timerequired for data collection. A number of systems have been implemented employing custom-made concave arrays 6, 7, 9, 13, 26, 27 , whileconventional linear arrays developed for medicalultrasoundare commercially available and attractive for optoacoustic imaging and tomography $16,28,30,35,36$. Medical ultrasound linear arrays are transducer assemblies with rectangular elementsarranged in a line with a fine spatial sampling,and are typically available for central frequenciesranging from 1 to $20 \mathrm{MHz}$ with bandwidth as high as $60-80 \%{ }^{37}$. Such detectorsare typicallybuilt with a cylindricalacoustic lens which enhancessignal detection around the median planeof the array $^{33}$ and provides a large depth of focus ${ }^{38}$. For optoacoustic tomography, the inplanespatialresolution perpendicularly to an ultrasound array (axial resolution)is primarily defined by the bandwidthof the detectors, whereas the lateral resolution (along the array) is limited by the effective angular aperture of the array. Due to the length of a typical linearultrasound array and the typically small size of an element along this length ${ }^{39}$-that provide a large angle of acceptance-, conventional linear arrays offer lateralresolutions in the order of a few tens to hundreds of microns, depending on the operating frequency. Conversely, the detector focus along the perpendicular (transverse) dimensionis not sufficient to achieve similar resolution; instead the resolution in thetransverse dimension is at least an order of magnitude worse than in the lateral dimension. Linear scanning of the array perpendicularly to the imaging plane has been used to cover a planar detection surface, and volumetric images have been formed by stacking $2 \mathrm{D}$ images ${ }^{16,30,35}$. However, because of the small angular aperture due to the fixed cylindrical focus, the resulting volumetric images have poor resolution along the scanning axis. The lateral resolution was shown to improveby rotating the $\operatorname{array}^{28,36}$, the rotation axis being perpendicular to the imaging plane. However, the limited view issues along the rotation axis remained unsolved.

Despite the limitations in imaging performance achieved so far, commercially available ultrasound arrays are attractive detector assemblies for optoacoustic imaging. To extend the performance achieved by conventional linear arrayswhen employed in the context of three-dimensional optoacoustic tomography, we investigate in this paper a novel scanning geometry. The proposed geometry capitalizes on the large aperture of the array to achieve good resolution along the length of this array while it employs two movements of the detectors, i.e. translation and rotation, to enclose the imaged object 
andachieve high resolution imaging along the other two geometrical dimensions. This scanning geometry

97 resembles the translate-rotate scanning method for the first generation of computed tomography x-ray

98 imaging $^{40}$. The capability of this geometry to provide high resolution three-dimensional optical images of

99 large volumes is demonstrated here with a commercial 128 element linear array, which is used in

100 ultrasonography at 5.0/7.0 MHz. The system is first characterized with phantoms, and its performance is

101 then shown on ex vivo mice.

\section{II. MATERIALSAND METHODS}

\section{A. EXPERIMENTAL SETUP}

The experimental setup used in this study is shown in Figure 1. The optoacoustic system consists of three main components: the illumination part comprising a nanosecond pulsed laser and a fiber bundle, the ultrasound detector and its data acquisition system, and the array holder with motorized translation and rotation. The fiber bundle ends, the ultrasound transducer and the sample wereimmersed in a water tank to ensure acoustic coupling. The tank was filled with a home-made isotonic saline solution $(0.90 \%$ $\mathrm{w} / \mathrm{v}$ of $\mathrm{NaCl}$ in deionized water) to preserve the ex-vivo samples. The solution was stabilizedat room temperature.

The excitation light originated from a tunable $(690-900 \mathrm{~nm})$ optical parametric oscillator laser (Phocus II,Opotek Inc., Carlsbad, California), delivering $<10$ ns duration pulses with a repetition frequency of $10 \mathrm{~Hz}$. The beam was guided into a silica fused-end fiber bundle (CeramOptec GmbH,Bonn, Germany) consisting of 640 fibers partitioned into 4 legs. The legs were positioned $5 \mathrm{~cm}$ away from the sample to create an illumination pattern of $\sim 15$ mmheight and $\sim 20 \mathrm{~mm}$ width on the surface of the sample. The illumination was fixed and one-sided. A12 $\mu \mathrm{m}$ thick aluminum foil, shaped as a half hollow cylinderof radius $\sim 15 \mathrm{mmand}$ height $10 \mathrm{~cm}$ (figure1(a), not represented on Figure 1(c)), was positioned around the sample opposite to the illumination, and acted as an optical mirror to reflect diffused andstray light back to the sample.The foil was determined experimentally to be transparentfor the ultrasound frequencies considered here by comparing the broadband signals measured through water from an optoacoustic source with and without the foil (maximum one-way insertion loss of $2 \mathrm{~dB}$ at $12 \mathrm{MHz}$ ). The optical excitation was performed for all the experiments at a single wavelength: $760 \mathrm{~nm}$, and with a per- 
124 pulse energy at the laser output of $\sim 70 \mathrm{~mJ}$.This wavelength ensures a good penetration in biological tissue 125 and a contrast to deoxygenated hemoglobin ${ }^{41}$.

126 Ultrasound detection was performed with a 128-element linear array designed for small parts and 127 vascular ultrasonography imaging:Acuson L7 (Siemens Healthcare). The width of one element is $270 \mu \mathrm{m}$, 128 with a kerf of $30 \mu \mathrm{m}$ (i.e a pitch of $300 \mu \mathrm{m}$ ), and itsheight is $4.0 \mathrm{~mm}$. The elements are cylindrically 129 focused using an acoustic lens. The focal length was determined experimentally to be around $19 \mathrm{~mm}$. The 130 fixed f-number of the probe is therefore $\sim 4.75$, which can be considered as a weak focus. The probe has 131 been designed to be used in ultrasonography at $7 \mathrm{MHz}$ and in Doppler mode at $5 \mathrm{MHz}$. The array was 132 covered with an acoustically-transparentmetallized Mylar film to avoid direct illumination of the 133 elements. The detected signals weredigitized at $40 \mathrm{MS} / \mathrm{s}$ and with a 12 -bit resolution over a $16 \mathrm{mV}$ range, 134 witha 128 channelcustom built data acquisition system.

135 The linear array was mounted on two motorized stages: a rotarystepper motor (PRM1/MZ8, 136 Thorlabs GmbH, Karlsfeld, Germany) and a linear translation stage(MTS50/M-Z8, Thorlabs GmbH, 137 Karlsfeld, Germany). The stages were hanged from a platform above the water tank, and arranged in 138 order that the translation stage couldbe rotated.The linear array was mounted on the linear translation 139 stage so that its median plane was perpendicular to the translation axis of the stage.

140 The data acquisition system and the motors were computer-controlled. The following sequence 141 was used for the acquisition. The laser was run continuously at $10 \mathrm{~Hz}$ and triggered the acquisition 142 system.For each detector position, ten acquisitions were averaged and the result stored in the 143 computerbefore advancing to the next position. Averaging was employed to increase the signal-to-noise 144 ratio by averaging noise and laser fluctuations. 

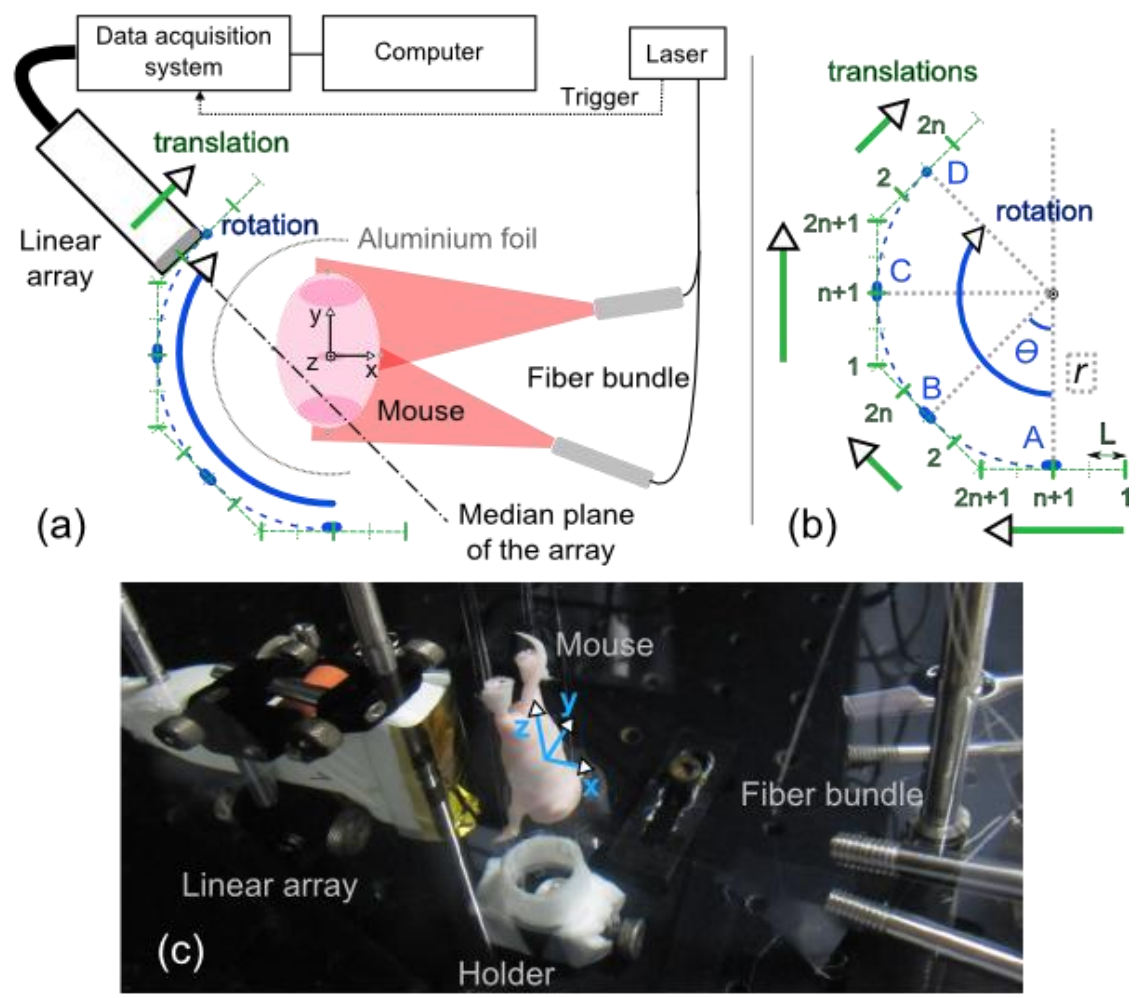

Figure 1 Experimental set-up shown here for the acquisition performed ex vivo on a mouse. (a) Schematic top view of the set-up. A Cartesian coordinate system is specified. The origin of the system is set so that the plane $z=0$ correspond to the middle of the linear array, and the $x$ - and $y$-axis so that the axis of the rotary stage corresponds to the $z$ axis. The z-axis corresponds to the elevation direction.(b) Schematic description of the scan geometry shown here for 4 rotary positions. The different positions of the array are presented. The positions of the rotary stage are indexed with capital letters while the positions of the translation stage are indexed with numbers from 1 to (2n+1). (c)Annotated picture of the experimental set-up.

The detector array was moved to discrete positions along the contour of a polygon (Figure 1 (b)).

157 Each of the linear segments of the contour (polygon sides) wastangent to a circle centered on the axis 158 ofrotation. The radius $\mathrm{r}$ of this circlewas set to be approximately equal to the focal length of the array $(\mathrm{r} \approx$ $15919 \mathrm{~mm}$ ), and was more accurately determined by imaging a calibrated phantom (see D). For the sake 160 ofsimplicity, thelinear segmentswerechosen symmetric with respect to the perpendicularradiiof the circle, 161 and the anglesbetween two consecutive radii had all the same measure $\theta=1.5^{\circ}$. The two extreme radii of the 162 polygonal contour define the angle coverage in azimuth, and made an angle of $178.5^{\circ}$, corresponding to 
$180^{\circ}-\theta$. This angle coverage hadtwo main advantages over the full anglecoverage $\left(360^{\circ}-\theta\right)$. First, the opening in the contour allowed setting a fixed and broad illumination of the object for all the positions of the array,i.e. an identical optoacoustic source. Second, it enabled to divide the total number of positions of the array by a factor of two, while fulfilling the 'visibility' condition in the xy plane ${ }^{31}$. On the condition that the lengths of the linear segments exceed the sizeof the imaged sample, the contour entirely contained the samplein the xy plane. The length of the polygonsideswas chosen here to bea multiple ofL $=750$ $\mu \mathrm{m}$ (Figure 1 (b)). This elementary length corresponds to the full width half maximum(FWHM)of the sensitivity fieldat $9.5 \mathrm{MHz}$ calculated for the fixed cylindrical focus of the array ${ }^{38}$. The maximum length of the polygon sides was chosen to be $13.5 \mathrm{~mm}$, and thereforecomprised 18 elementary segments of length L (corresponding to $n=9$ on Figure 1(b)). Theendpoints of these elementarysegments defined in this case 19 positions along a polygon side $(2 \mathrm{n}+1$ on Figure $1(\mathrm{~b}))$. To limitthe total number of measurements and then reduce the acquisition time, the detector positions were actuallyspaced by 2.Lfor each polygon side, and complementary patterns were chosen for two consecutive polygon sides. With the chosen angular step, this approach was determined to have no noticeable influence on the image resolution when compared todetector positionsspaced by L for everylinear segment.

The scan geometry was practically implemented by using the following motion sequence. The rotary stage was moved toa total of 120 positions, with a $\theta=1.5^{\circ}$ angular step.Between each rotation step (named by a capital letter in the schematic of Figure 1), the array was scanned linearly along the tangent to the circle by moving the translation stagein $2 . \mathrm{L}=1.5 \mathrm{~mm}$ steps, respectively to $\mathrm{n}=9$ or $\mathrm{n}+1=10$ translation positions.A total of 1140 positions of the array were covered per scan, for atranslationrange of 2.n.L $=13.5 \mathrm{~mm}$. The total acquisition time per scan was 1.5 hours with 10 time-averages on the signals.

\section{SAMPLE PREPARATION}

\section{PHANTOMS FOR CHARACTERIZATION AND CALIBRATION}

Turbid phantoms mimicking the optical scattering properties and the speed of sound of soft tissueswere prepared by mixing $2 \% \mathrm{w} / \mathrm{m}$ agar gel (Agar for microbiology, Fluka analytical) with 6\%v/v intralipid-20\% (Sigma). The gel was poured in cylindrical molds: $16 \mathrm{~mm}$ in diameter and $5 \mathrm{~cm}$ in height.Different calibrated absorbers were inserted in the gel for the characterization of the system.

The first phantomcontained a single black polyethylene microsphere of $200 \mu \mathrm{m}$ in diameter (BKPMS 180-212um, Cospheric, Santa,Barbara, CA).This phantomenabled us to determine an average 
speed of sound (dependent on the temperature in the water tank), and the value of the radius r(Figure 1).A

194 second phantom was prepared with $200 \mu \mathrm{m}$ microspheres. The microspheres were randomly spread over a

195 cross section of the phantom, around $1 \mathrm{~cm}$ away from the top. A similar and third phantom was prepared

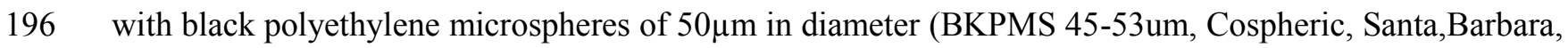

197 CA).A fourth phantom contained a black nylon suture of size 7-0 (diameter $\sim 50 \mu \mathrm{m}$,Ethilon Monofilament

198 Polyamide 66, Ethicon, Inc.) arranged in a cross along the length of the cylinder. The phantoms were

199 placed in the tank so that the axis of the cylinder corresponded approximately to the z-axis, and the 200 middle of the absorbers to the plane $\mathrm{z}=0$.

\section{ANIMALS AND HANDLING}

Two CD1® mice (Charles River Laboratories, Research Models and Services, Germany GmbH), four days old and seven days old respectively, were euthanized and imaged ex-vivo. Mouse husbandry, handling and euthanasia were performed according to the institutional and Bavarian government regulations in frame of approved animal protocol. Hair was removed with a hair removing lotion(Veet, Germany), and the mice were placed on acustom-made holder made of nylon wires tightened between two hollow cylinders of $16 \mathrm{~mm}$ in diameter. Nylon wires were chosen to limitthe interference of the holder withultrasound wave propagation. The mice were positioned so that their anteroposterior axis follows the $\mathrm{z}$ axis with their head towardsthe negative $\mathrm{z}$-direction, and with the illumination on their dorsal side (Figure 1). Different body parts were centered on the xy plane:the head for the younger mouse, and the abdomenfor the older one.

\section{IMAGE RECONSTRUCTION}

The recorded signals were first deconvolved from the electrical impulse response of the detection systemusing the Wiener filter algorithm. The impulse response was recorded for one element of the array as described by Rosenthal et al ${ }^{42}$ using an optoacoustic point source (focused light on a thin absorbing layer) located along the focal line in front of the element. The deconvolution process compensated for the frequency-dependent response of the sensor and signals with frequencies up to $12 \mathrm{MHz}$ could be recovered with good signal-to-noise ratio (SNR).Afterwards, the deconvolved signals were band-pass filtered(Butterworth, order 3) between $100 \mathrm{kHz}$ and $12 \mathrm{MHz}$ for noise removal. . 
Volumetric images were reconstructed on a rectangular cuboid Cartesian grid centered on the measurement coordinate system (Figure 1). The grid dimensions were adapted to the sample size, and the voxel dimensions were set to $\Delta \mathrm{x} \cdot \Delta \mathrm{y} \cdot \Delta \mathrm{z}=37 \mu \mathrm{m} \cdot 37 \mu \mathrm{m} \cdot 74 \mu \mathrm{m}$ to achieve sufficient spatial sampling with regard to the expected in-plane (xy plane) and elevation (along z-axis)resolutions. A filtered backprojection algorithm was used in this study to reconstruct the volumetric images. Several modifications were performed to the backprojection algorithm proposed by $\mathrm{Xu}$ and Wang ${ }^{43}$ for cylindrical geometries. The phenomenological description of the algorithm given in ref ${ }^{2}$ was 230 consideredto adaptthe algorithm to the specificity of the detector.First, only the first time-derivative of the

231 processed signal : $-\frac{\partial V}{\partial t}$ was backpropagated onto the image grid.The non-derivative part of $\mathrm{Xu}$ and

232 Wang's formula : $\frac{V}{t}$ was found negligiblewith respect to the derivative term, most likely because of the 233 distance between the source and the detectors ${ }^{2}$. Withthe backprojection algorithm, the value of each point 234 of the image can be assigned independently. The reconstruction of theimages was then performedusing all 235 the detection signals, but for2D grids at constantz independently. The 2D images were then stacked to 236 form the volumetric image. This approach has the advantage that a dynamic aperturereconstructioncould 237 be implemented along the $\mathrm{z}$ axis in order to keep the elevation angle of acceptance constantfor all the 238 points of the 3D image.Dynamic aperture reconstructionis a standard feature forconventional ultrasound 239 linear detector arrays ${ }^{44}$. It was performed for each position of the arrayindependentlyby adaptingthe 240 receiving aperturetothe depthuntil the maximum physical aperture of the array was reached. For each 2D 241 grid, thegrowing apertures were centered on its $\mathrm{z}$ coordinate. The ratio of the depth to the aperture 242 length(i.e. the f-number in receive) was set to 0.5 here.Dynamic aperturealso compensates the geometrical 243 spreading (or diffraction attenuation) of the ultrasonic wave. In addition of being dynamic, the aperture 244 was also apodizedto avoid strong side lobes. A window function with a half-sine windowon each side(for $24520 \%$ of the aperture on each side) and flat otherwise was implemented.This implementation aimed at 246 apodizing the aperture sides since other physical apodization of the signals also occursduring the 247 detection process,particularly due to the finite size of the detectors.

For accurate reconstructions, we experimentally determined the average speed of sound in the 249 medium cand the radius $\mathrm{r}$ (Figure 1(b)). The speed of sound was determined by measuringthe first 250 phantom for a single position of the array. The microsphere was then placed in the median plane of the 251 array.Using the positions of the array elements specified by the manufacturer, the first time derivative of 252 the processed signals was backpropagated, on a 2D gridincluding the microsphere, assuming different 
speeds of sound. The amplitude of the image was maximized for $c=1480 \mathrm{~m} \cdot \mathrm{s}^{-1}$, whichwasthus considered as the speed of sound in the medium.To determine the value of the radius $r, 3 \mathrm{D}$ reconstructions with different $r$ values were computed for a scan acquisition of the first phantom. The value that maximized the amplitude of the 3D image was determined to be $\mathrm{r}=19.25 \mathrm{~mm}$ and was set as a parameter for subsequent reconstructions.

The images obtained with thefiltered backprojection algorithm comprised noise and non-physical negative values ${ }^{45}$ and were processed before $3 \mathrm{D}$ visualization.Image processing was based on the assumption that the majority of the reconstructed voxelswere comprised onlybynoise, since the actual absorbing targets occupy only a small part of the total volume in the region of interest. With this assumption, the following postprocessing routine was developed. First, a histogram of the image values was computed. Voxels with values below the one corresponding to the maximumof the histogram were disregarded. This step allows removing the voxels with nonphysical values, mostly negative ones. For the remaining voxels, the first $0.02 \%$ was disregarded to limit the noise from being displayed, and the last $0.02 \%$ to $0.1 \%$ was saturated to improve the image visualization. The $3 \mathrm{D}$ images were visualized using maximum amplitude projections and a rotation around one axis. This visualization was performed withthe 3D project option of ImageJ.

\section{RESULTS}

\section{A. INFLUENCE OF THE NUMBER OF TRANSLATION POSITIONS}

Four different reconstructions of the data acquired for the second phantom (sparse $200 \mu \mathrm{m}$ microspheres)were performed. For each reconstruction, a different subset of the 1140 positions of the array was selected to artificially vary thetranslationrange.The positions considered for each of the subsets respected the symmetry to the radii of the circle, and the number of rotary positions and the translation step between positions on a linear segmentwere kept constant and respectively equal to 120 and $1.5 \mathrm{~mm}$. Only the number of translation positions was changed, anddatasets corresponding to translationrangesequal to $1.5 \mathrm{~mm}, 4.5 \mathrm{~mm}, 9 \mathrm{~mm}$ and $13.5 \mathrm{~mm}$ wereformed. The corresponding total numbers of translations positions for two successive linear segments were respectively: 3 (corresponding to $n=1$ on Figure $1(b)), 7(n=3), 13(n=6)$ or $19(n=9)$.

An optical picture of the absorbers embedded in the phantom and the maximum-amplitude projection (MAP) optoacoustic images along the z-axis corresponding to the four reconstructions are 
presented in Figure 2. For translationrangesmaller than the effective diameterof the phantom (Figure 2 (b)-(d)), the microspheres located in the vicinity of the rotation axis, within acircle ofdiameter slightly

284 larger than the travel range plus $\mathrm{L}=750 \mu \mathrm{m}$ (to account for the thickness of the focal spot), appear with a 285 round shapeon the MAP images. However, for the microspheres located further away from the rotation 286 axis, the lateral resolution along the azimuthal direction reduces with the radial distance. This 287 smearingeffect can be interpreted as a limited view effect due to the directivity of the detectors.For the 288 translationrangematching the size of the phantom, all the microspheres of the phantom can be identified 289 and were reconstructed with a round shape on the MAP image (Figure 2 (e)). An amplitude gradient can 290 be seen along the $\mathrm{x}$ direction, due to the one sided illumination and optical scattering. thein-plane resolution has a two-dimensional isotropy and homogeneity. With the scan geometry proposed here, the translationrange can therefore be adjusted to fully contain theimaged object.

(a)
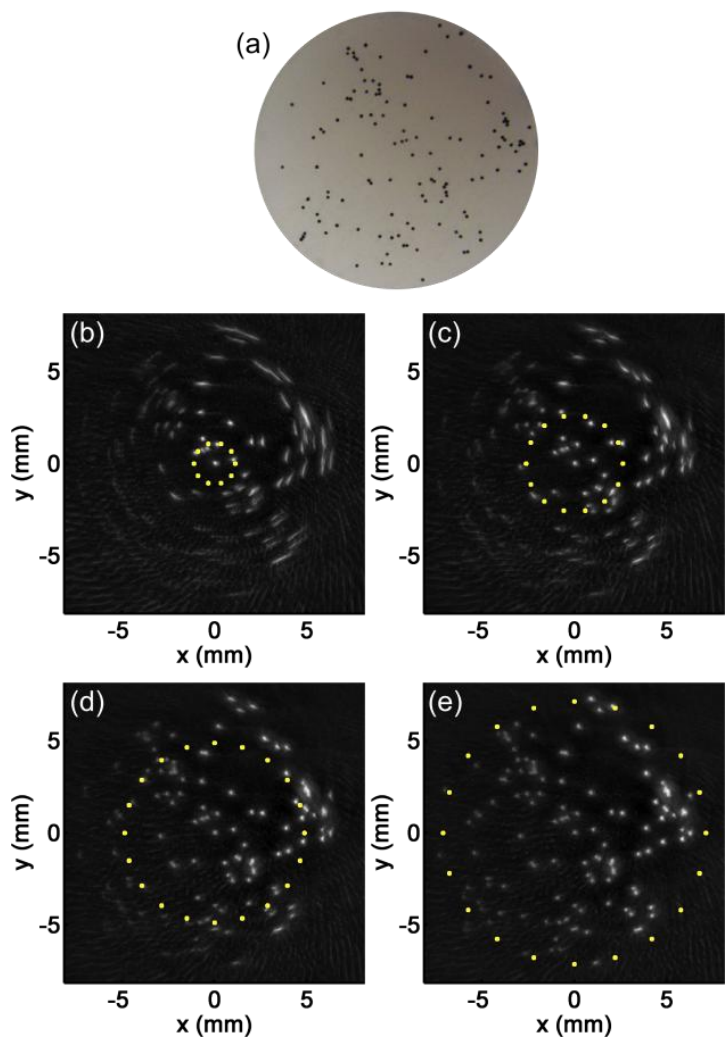

Figure 2 Reconstructionswith different translationrangesof the second phantom(comprising $\varnothing 200 \mu \mathrm{m}$ microspheres randomly spread over a cross section). (a) Optical picture showing the spatial distribution of the absorbers. (b)-(e) Maximum-amplitude projection optoacoustic images along the z-axis for translationranges of: $1.5 \mathrm{~mm}, 4.5 \mathrm{~mm}, 9.0$ 
$\mathrm{mm}$ and $13.5 \mathrm{~mm}$ respectively. The yellow dottedcircles have a diameter equal to the translationrange plus $\mathrm{L}=750 \mu \mathrm{m}$ to account for the thickness of the focal spot.

\section{B. Resolution IN 3D}

The three-dimensional resolution of the system was investigated by imaging absorberswhichpeak frequency is expected to be greater than the highest recorded frequency ${ }^{46,47}$ : $\varnothing 50 \mu \mathrm{m}$ microspheres and threads.

\section{RESOLUTION OF ISOTROPICOBJECTS}

The third phantom,comprised of $50 \mu \mathrm{m}$ microspheres randomly spread over a cross section, was imaged with the system. The MAP image along the $\mathrm{z}$ axis of the entire phantom is presented in Figure 3 (a), and shows that the microspheres could be reconstructed over the entire cross section. Besides amplitude variations that could partially be attributed to the inhomogeneity of the light fluence, and a thresholding of the brighter voxels, no loss of azimuthal resolution with the distance to the rotation axis is noticeable.

The $3 \mathrm{D}$ resolution of two microspheres located at a distance of $\sim 5 \mathrm{~mm}$ from the rotation axis and towards the open bound of the scan trajectorywas analyzed. A cuboid containing the two microspheres $(15 \cdot 15 \cdot 11$ voxels)was extracted from the 3D image (green square, Figure 3(a)). The maximum amplitude projections of this cuboid in the three orthogonal planes are displayed in Figure 3 (b)-(d), and the normalized and centered profiles of two of these MAP images are plotted in Figure 3 (e). Only the nonphysical negative values were disregardedto represent these images. The MAP images show that,along the three directions, the two microspheres are resolved identically. The FWHM dimensionsof one of the sphereswere determined to be: $140 \mu$ min-plane and $327 \mu \mathrm{m}$ in elevation (Figure $3(\mathrm{e})$ ). The discrepancy between the two resolutions could beattributed to the limited view in elevation as opposed to the full enclosure in-plane.

In addition to the two microspheres, the resolution was analyzed along the width of the phantom. A volumetric sliceperpendicular to the $y$-axiswas selected.Its width was chosen so that microspheres are spread all over thewidth of the phantom but with distinct $\mathrm{x}$ coordinates. The MAP images of the slicealong the z-axis and the y-axis are shown in Figure 4 (a) and (b). The FWHM values of the MAP amplitude profiles along the $\mathrm{y}$ axis and $\mathrm{z}$ axis were determined for 23 distinct microspheres,and compared to the values found previously (Figure 4 (c)). The results confirm that the in-plane and elevation resolution are approximately constantover the width of the phantom, and consistent with thevalues found for the two microspheres of Figure 3(b)-(e). The FWHM of the amplitude profiles along the y axis was 
330 the microspheres was observed to have a small angle with the xy plane.
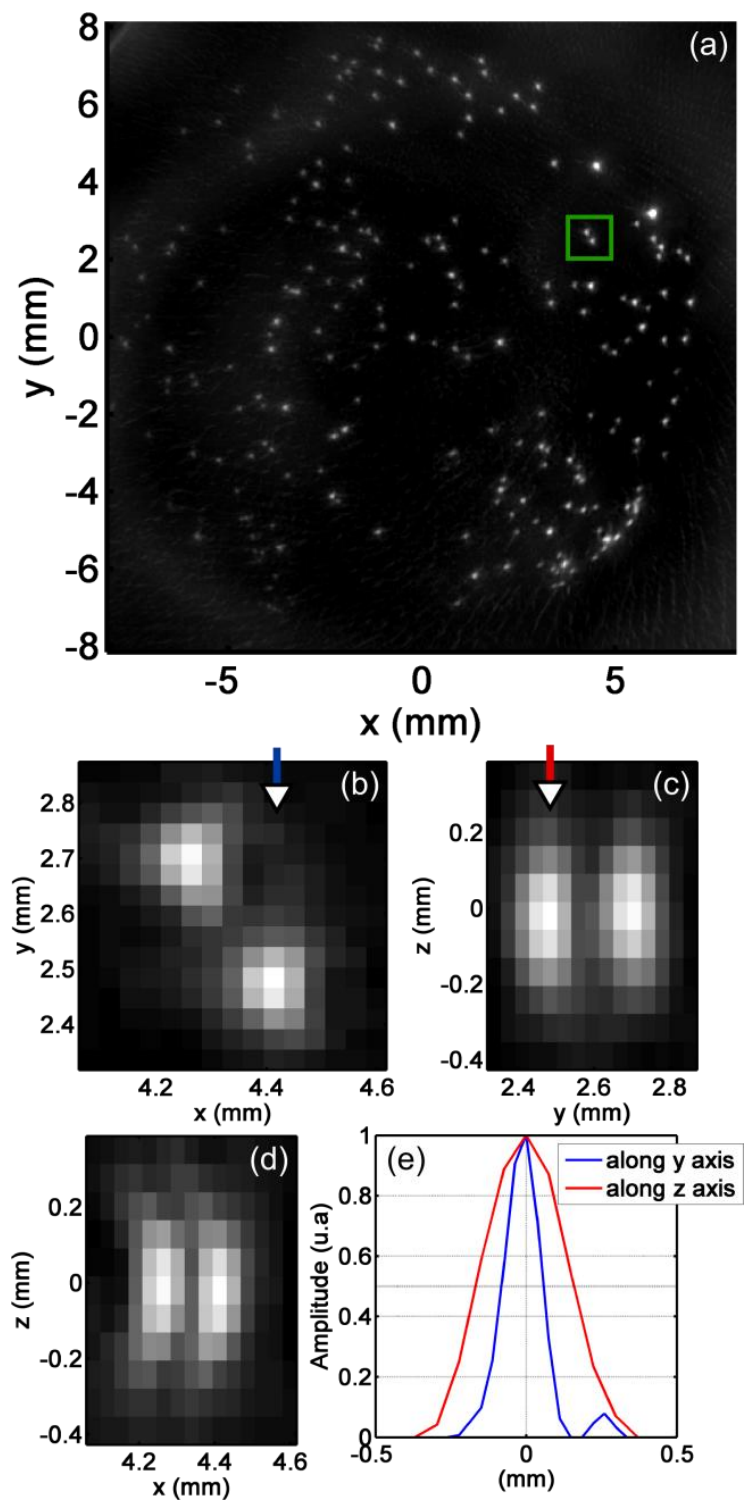

Figure 3Reconstructions of the third phantom (comprising $\varnothing 50 \mu \mathrm{m}$ microspheres randomly spread over a cross section). (a) MAP image of the entire region-of-interest along the z-axis. The green square indicates the limits of a subregion of interest. MAP images of this sub region along (b) the $z$-axis, (c) the $x$-axis, and (d) the $y$-axis. (e) Amplitude profile of the lines marked with an arrow on (b) and (c). The amplitude profiles were normalized and the peak valueswere centered. The FWHM of the profile along the $y$ axis is $: 140 \mu \mathrm{m}$ and along the $\mathrm{z}$-axis $: 327 \mu \mathrm{m}$. 


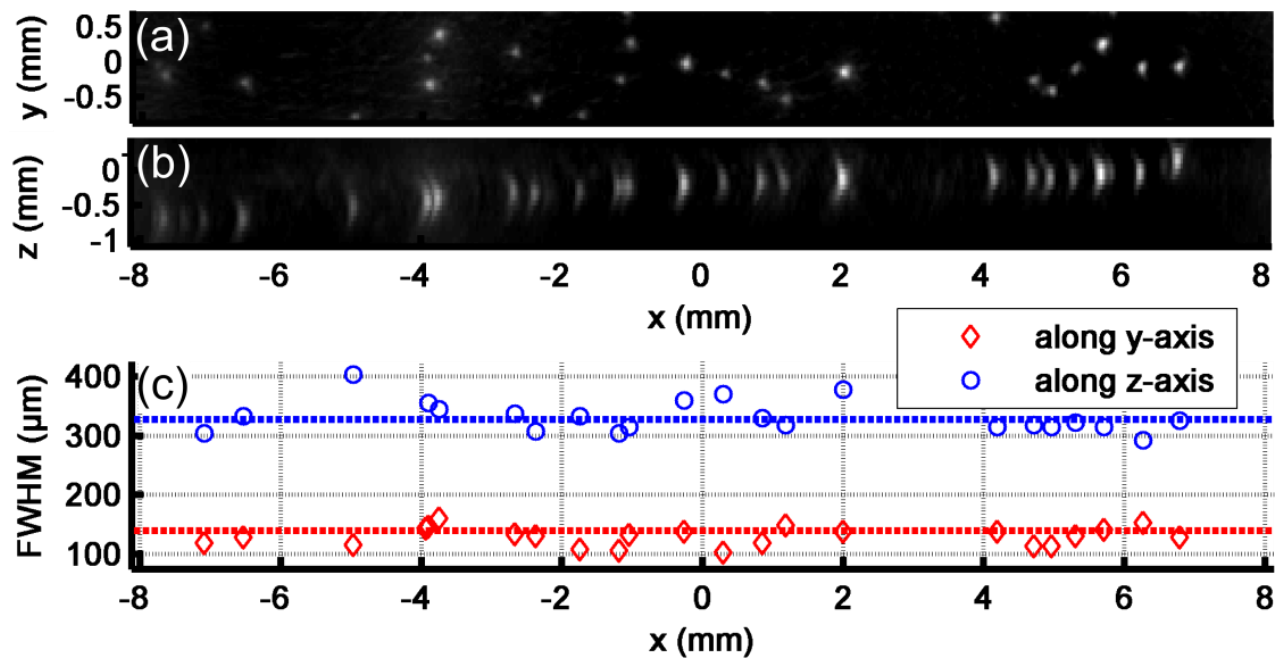

Figure 4Reconstructions of a volumetricslice of the third phantom,perpendicular to the $y$ axis(a) MAP image along the z-axis. (b) MAP image along the $y$-axis. (c) FWHM of the amplitude profile along the $y$ axis and $z$ axis of 23 distinct microspheres distributed along the length of the slice. The dashed lines mark the value found for the microsphere of Figure 3.

\section{RESOLUTION IN TERMS OF SEPARATION BETWEEN 2 OBJECTS}

The fourth phantom,comprised of a cross from a $\varnothing 50 \mu \mathrm{m}$ thread, was imaged to study the resolutionin terms of ability to separate two objects. The MAP image along the y-axis is shown in Figure 5(a). Two slices orthogonal to the z-axis were selected from the volume and are presented in Figure 5 (b) and (c) respectively. The slices were not post-processed. The first slice (Figure 5 (b)) was taken well

347 above the cross intersection and shows the position of the threads in the xy plane. The threads appear with

348 a round shape on this slice: no elongation in the direction given by the projection of the thread in the xy 349 plane can be noticed. The second slice (Figure 5 (c)) was taken close to the location of the intersection of 350 the threads, and corresponds to theslice were the saddle pointbetween the individual reconstructions of the 351 threads first develops. The distance between the two maxima corresponds to the resolution in the sense of 352 the Sparrow resolution criterion.It is determined here to be $189 \mu \mathrm{m}$, which is similar to thein-plane 353 resolution determined with the previous phantom. As opposed to spherical objects, however, the elevation 354 resolution interferesdue to the absorber extension along the z-axis. The $3 \mathrm{D}$ visualization of the thread 355 cross is presented on Video 1. 

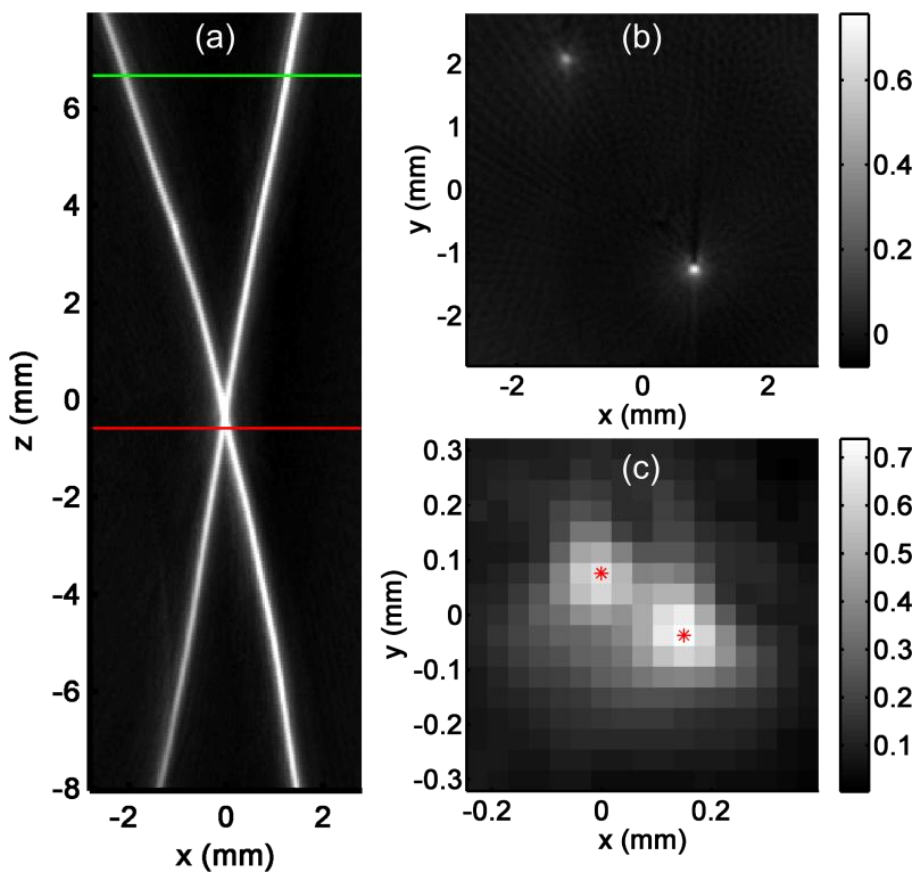

Figure 5Reconstructions of the fourth phantom (comprised of cross from a $\varnothing 50 \mu \mathrm{m}$ thread). (a) MAP image of the entire region-of-interest along the $y$-axis. (b) slice corresponding to the plane marked with a green line on (a). (c) slice corresponding to the plane marked with a red line on (a). The star markers indicate the position of the maxima corresponding to the reconstruction of each thread. The distance between the two stars is $189 \mu \mathrm{m}$. For the two slices (b) and (c), the images were not postprocessed but the voxel values were normalized by the maximum value of the 3D image, the brightest voxel being situated at the intersection of the cross.

Video 1: This video corresponds to Figure 5(a), and shows the 3D optoacoustic volume image of the thread cross. Rotating maximum amplitude projection images around the $\mathrm{z}$ axis are displayed with $2^{\circ}$ angle between the projections, and at a frame rate of $\mathbf{1 5}$ images per second

\section{EX-VIVO ANATOMIC IMAGES OF MICE}

\section{ABDOMEN}

The abdomen of the 7 day old mouse was imaged ex-vivo. The MAP image along the $\mathrm{x}$-axis is presented in Figure 6 (a). This image corresponds to the frontal projection. It is displayed here so that the mouse legs are located at the bottom of the image, and the left side of the mouse is on the left of the

372 image (as if the mouse was seen from its back).Anatomical structures can be visualized,in particular the

373 kidneys, the spleen, a partial lobe of the liver, and the femurs (bone marrow). Several major vessels as the 374 abdominal aortaand the vena cava could be identified, but multitude of smaller vessels can be seen as well, especiallyin the 3D visualization (Video 2) and in single slices (Figure 6(b)-(e)). Four different 
slices on the upper region of the abdomen are displayed in Figure 6(b)-(e). The slices were not postprocessed, and negative values can therefore be seen. The presence of these negative values can shadow some structures such as the abdominal aortaand the vena cava for $\mathrm{z}<-4 \mathrm{~mm}$ (Figure $6(\mathrm{a}-\mathrm{c})$ ).
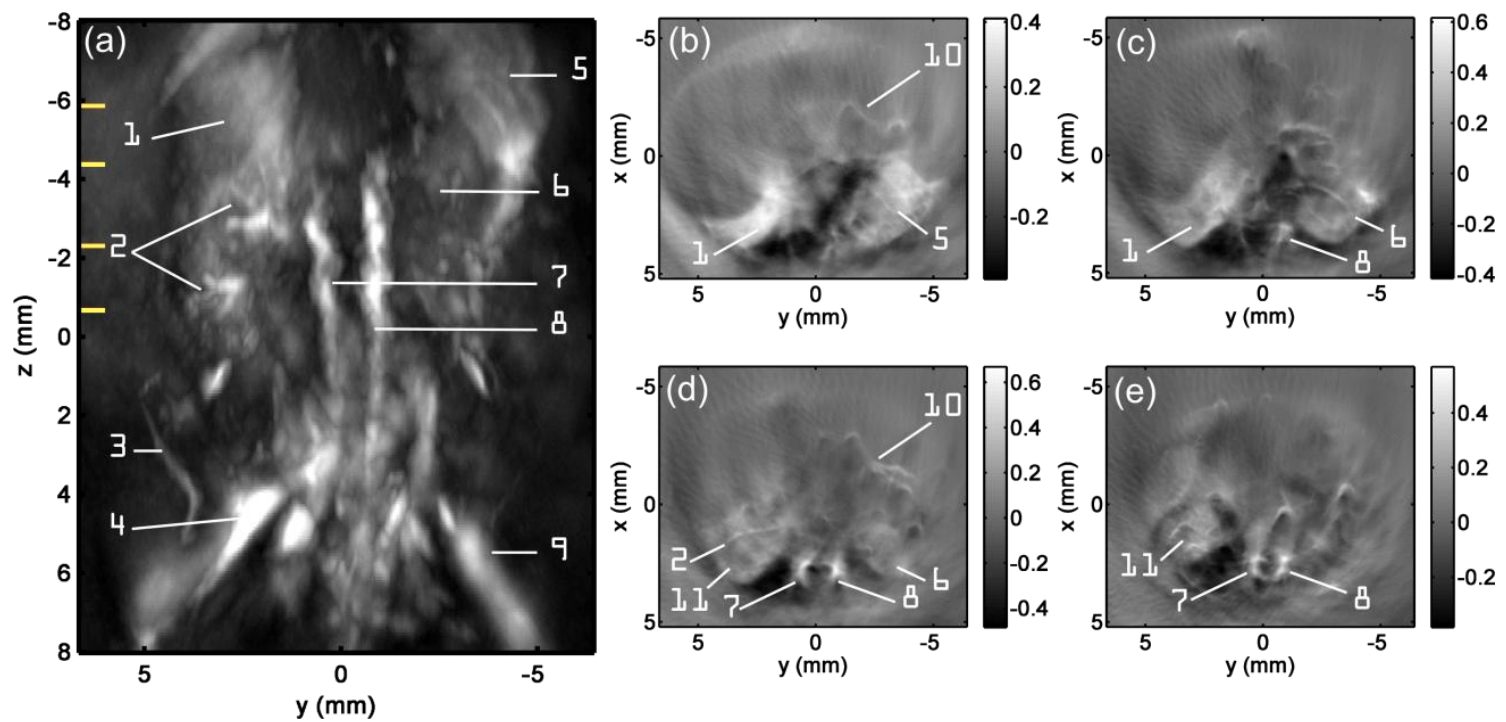

Figure 6Reconstructions of the abdomen of the 7-day-old mouse. (a) MAP image of the entire region-of-interest along the $\mathrm{x}$-axis. The slices (b)-(e) correspond to the yellow marks on the side of (a), and are ordered by increasing $\mathrm{z}$. For each slice the images were not post-processed. The voxel values are normalized by the maximum value of the $3 D$ image. Legend: 1. Spleen, 2.Interlobar vessels of the left kidney, 3.Ischiatic vein, 4. Saphenous vein, 5.Partial lobe of the liver, 6.Right kidney, 7. Abdominal aorta, 8. Vena cava, 9. Femur, 10. Intestine vessels,11. Left kidney. The anatomical features were correlated with published mouse anatomy ${ }^{48}$.

Video 2: This video corresponds to Figure 6 (a), and shows the 3D optoacoustic volume image of the abdomen of the 7-day-old mouse. Rotating maximum amplitude projection images around the $z$ axis are displayed with $2^{\circ}$ angle between the projections, and at a frame rate of 15 images per second.

\section{HEAD REGION}

The head of the 4 day old mouse was imaged ex-vivo. The MAP images along the x-axis and the z-axis areshown respectively in Figure 7(a) and (b). The head is oriented so that the mouse snout points towards the negative z-direction. The vascular anatomy of the head can be visualized on these two projections, and in particularthe dural venous sinuses and the supraorbital veins (Figure 7). The different

394 parts of the head can be clearly identified and many fine structures can be visualized on Video 3 . Three 395 coronal slices were selected (Figure 7 (c)-(e)) to better show vascular structures hidden on the MAP 396 projection because of the brightness of the superior sagittal sinus. This brightness can be explained by 

the structures of the neck including the jugular veins.
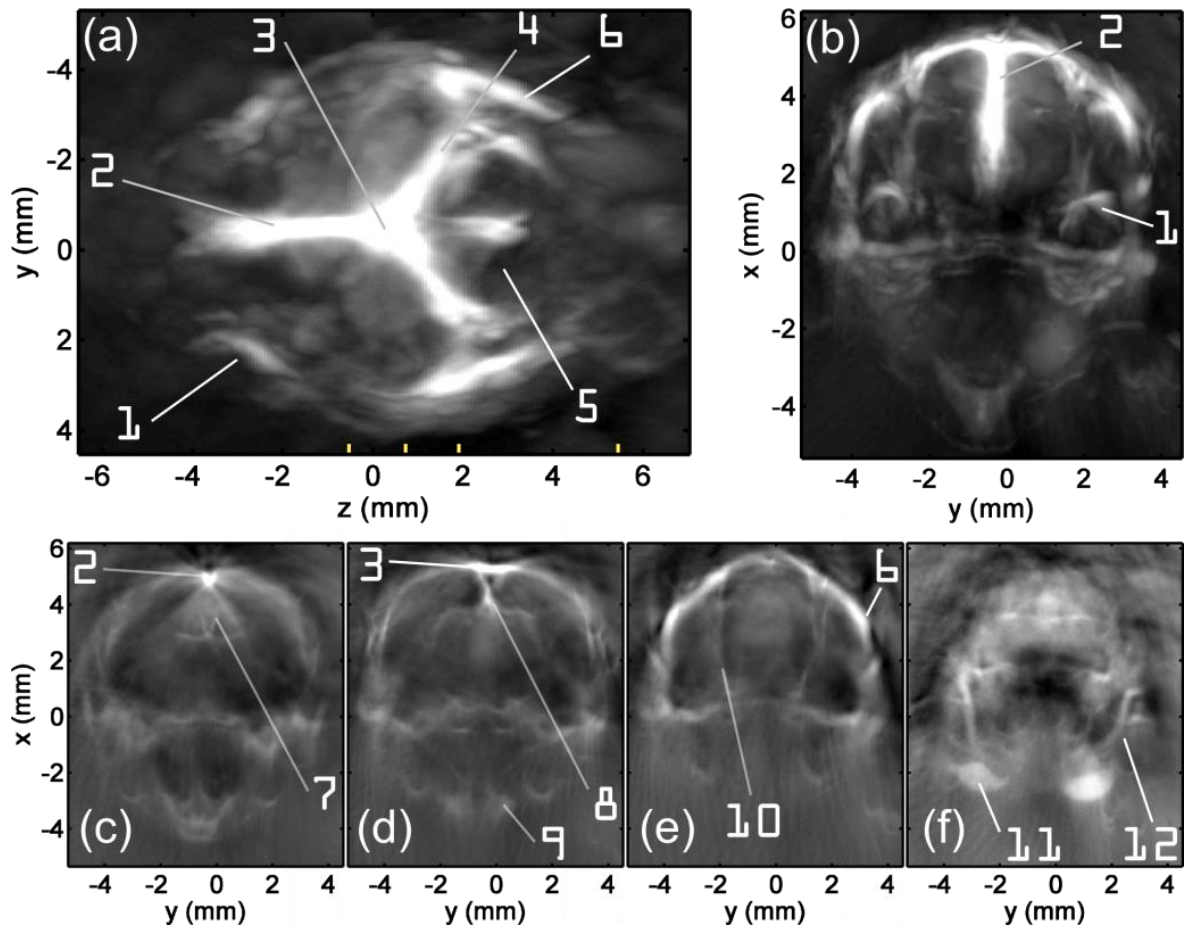

Figure 7Reconstructions of the head of the 4-day-old mouse. MAP image of the entire region-of-interest (a) along the $x$-axis, (b) along the z-axis. The slices (c)-(f) correspond to the yellow marks on the bottom of (a), and are ordered by increasing z. Legend: 1. Supraorbital vein, 2. Superior sagittal sinus, 3. Confluence of sinuses, 4.Right transverse sinus, 5.Cerebellum , 6. Temporal vein, 7. Inferior sagittal sinus, 8.Straight sinus, 9. Facial vein, 10. Sigmoid sinus, 11.Jugular vein, 12. Maxillary vein. The anatomical features were correlated with published mouse anatomy ${ }^{48}$.

Video 3: This video corresponds to Figure 7(b), and shows the 3D optoacoustic volume image of the head of the 4-day-old mouse. Rotating maximum amplitude projection images around the $x$-axis are displayed with $2^{\circ}$ angle between the projections, and at a frame rate of 15 images per second.

\section{Discussion}

We examined in this study the performance of a novel detection geometry for volumetric optoacoustic tomography using a conventional linear array employed in ultrasound imaging. To make

411 optimal use of the detection characteristics of such a transducer, we investigated the role that translation

412 and rotation of the detector array in relation to the imaged object plays on the achieved image quality. The

413 study demonstratedthatthe proposed geometrical configuration provides a versatile system adapted to

414 perform whole-body small animal imaging. The arrangement allows theseamless adjustment of scan 
415 parameters to fit the radial dimensions of the region-of-interest within the sample.The translationrange

416 along the polygon sides was shown to define the radial distance for which no loss of in-plane resolution

417 along the azimuthal direction could be noticed(Figure 2).Conversely, the number of positions of the

418 detector array, and therefore the scanning time, can be adapted depending on the dimensions of

419 thesample.Two different mice and four phantoms were imaged in this study, which shows the

420 capabilitiesof the imagingsystem.

With a translationrange corresponding to the radial size of the sample, the in-plane resolution was

422 found to have two-dimensional isotropy and homogeneity over the width of the sample even for absorbers

423 emitting with peak frequencies higher than the low-pass cut-off frequency of the system ${ }^{46}$ (Figure 3 and

424 Figure 4). The isotropy and homogeneity of the in-plane resolution are a consequence of the scanning

425 geometry, in which the sample is entirely contained and probed with a high density of sensor positions. In

426 this manner, optoacoustic waves arising from the entire sample were captured at each rotation angle, and

427 the full-view criterion was fulfilledin-plane.Indeed, for objectsinsidethe circledefined by

428 thetranslationrange, normal vectors drawn onto alltheirin-planeboundary pointspass in one direction the

429 detection angular aperture. In-plane boundaries could therefore be recovered stably ${ }^{31}$. Conversely, because

430 of the limited angle of acceptance of the focused detector, in-plane limited-view issues occurfor objects

431 outsidethe circle, and theazimuthal resolution wastherefore degraded(Figure 2).The in-plane

432 FWHMdimensions for $50 \mu \mathrm{m}$ diameter microspheres werefound to be approximately $130 \mu \mathrm{m}$, for the

433 detection geometry and reconstruction algorithm selected in this study. The acoustic wavelength in the

434 medium corresponding to the low-pass cutoff frequency of the system $(12 \mathrm{MHz})$ is: $\lambda_{\mathrm{c}} \approx 123 \mu \mathrm{m}$, which

435 implies that the reconstructed in-plane FWHM dimension of the sphere and the optimal resolution

436 theoretically achievable $\left(0.8 \lambda_{\mathrm{c}} \approx 99 \mu \mathrm{m}^{5}\right)$ areon the same order of magnitude. The discrepancy between the

437 two values could first be attributed to the finite dimension of the microsphere. However, the sphere is a

438 solid which longitudinal speed of sound is larger than the one of the surrounding aqueous medium.As a

439 consequence, the sphere dimension cannot be simply subtracted to obtain the effective resolution of the

440 system. The finite size of detectorsmay also have an effect on the resolution, as the spatial averaging on

441 the detector surfacedistorts the signals detected for optoacoustic sources outside of the focal zone, and

442 shifts their frequency spectrum towards lower frequencies ${ }^{33}$. This effect, termed spatial impulse response

443 of the detector ${ }^{49}$,is not taken into account by the filtered backprojection reconstruction algorithm, which

444 assumes point-like detectors and non-distorted signals.The finite size of the detectors, theirfocusing

445 properties, and the discrete positions of the detector array could also partially explain the in-plane

446 amplitude variation between the reconstructed microspheres (Figure 3(a) and Figure 4(a)). The spatial 
averaging is indeed not equivalent for all the microspheres of the phantom when a translation scan is performed. This effect is however expected to be less significant for larger absorbers since their peak frequency is lower, which results in acorresponding focal zone with larger dimensions.A reconstruction algorithm able to take into account the spatial impulse response of the detector, such as in ref. ${ }^{33}$, would be needed to improve the in-plane resolution and amplitude homogeneity. Such investigations are beyond the scope of this paper, but will be considered in future studies.

Due to using a dynamic apertureadjustment, the elevation resolution was shown to be kept constant and uniform over a width of $15 \mathrm{~mm}$ (Figure 4), unlike circular tomography withcylindrically fixed focused detectors, which focal line is perpendicular to the axis of rotation ${ }^{23}$. The elevation FWHM dimension of microspheres of $50 \mu \mathrm{m}$ in diameter was found to be about $330 \mu \mathrm{m}$,i.e. more than twice the inplane FWHM value. Since the microspheres are isotropic absorbers, it can be concluded that the discrepancy between the in-plane and elevation resolution is due to the detection geometry. The main limitation of the proposed geometry in terms ofangular aperture is the finite aperture of the array along the elevation direction. The sample is indeed only partially enclosed in terms of the elevation angle. The angle of acceptance wasfurther reduced in order toobtaina uniform resolution in elevation. Finally, this resolution is degraded by the spatial averaging on the transducer surface, which effectivelycreates an apodization function, stronger at high frequencies. The elements of the array are indeed not infinitely thin but have a width of $270 \mu \mathrm{m}$, that is to say around $2 . \lambda_{c}$, which results in non-negligible spatial averaging for sources located at a steep elevation angle with respect to the sensor. Taking into account the spatial impulse response of the detector in the reconstruction algorithm would reduce this apodization and should then improve the elevation resolutionas well.

Despite the limitationsdue to the reconstruction algorithm and the limited view, the obtained images can be qualified as high resolution. The three-dimensional reconstruction of the cross (Video 1) and mice (Video 2and Video 3) show that small structures can be visualized and that their shape correspond to the implanted absorbers or the expected anatomy ${ }^{48}$. Moreover, no complicated image postprocessing was performed to obtain the $3 \mathrm{D}$ volume rendering, as opposed to the routines developed in other studies ${ }^{6}$. The negative and non-physical values that appear on the images (Figure 6) and that create some shadowing effects are most probably due to the non-optimal reconstruction algorithm, which is not adapted for finite-sized focused detector or limited solid-angle coverage.

For the implementation of the detection geometry used in this study, one full acquisition took 1.5hours with 10 time-averages on the signals, that is to say an average 4.8 seconds per position of the 
array.Since the acquisition of the signals took around 1 second per position (due to the $10 \mathrm{~Hz}$ pulserepetition rate of the laser), the time-consuming part was therefore the motion of the array. The current scanning is quite long for in-vivo experiments, however, several solutions can be considered to reduce significantly this scan duration. First,since the motion of the array is the most time consuming part of the acquisition, faster stepper motors could be used. Second, a continuous motion of the detector has been shown to reduce dramatically the acquisition time ${ }^{23}$ and could also be implemented for this detection geometry. On the other hand,the large number of positions used in this study could probably be significantly reduced. Using a reconstruction algorithm that takes into account the spatial impulse response of the transducers, better quality images are expected to be obtained with less sensor positions.

487 With the filtered backprojection algorithm, thespatial averaging of the optoacoustic waves on the detector 488 surface for optoacoustic sources outside of the focal zone of the array is indeed compensated by the large 489 number of positions, the signals originating from the focal zone being stronger than the distorted ones. Additionally, the fine translation step implemented here aimed at capturing the high-frequency components arising from the entire sample by translating the focal spot, so as to demonstrate the high resolution capability of the detection geometry. If the required resolution is lower than in the present study, the translation step-size could be accordingly adapted, which would result in shorter scanning duration.A systematic study of the influence of each scan parameters on the image quality is beyond the scope of this paper, but will be considered in the future to improve the acquisition time.Lastly, a higher repetition rate laser could also be used to accelerate the acquisition of the signals, and the number of time averages per positionoptimized in regards to the high number of individual measurements projections. interchangeability ofthe transducer array. No dedicated prototype was built, instead a conventional ultrasound array was used. A large range of linear arrayshave already been developed for ultrasonography, and are available commercially. Among the wide variety of length,number of elements, and central frequencies available, the high central frequency detectors, and the large aperture ones are of special interest for the proposed detection geometry. The combination of a large number of elements and a smallpitchwould indeed allow increasing the angular aperture in elevation.Commercially available linear arrays for medical ultrasound have as many as 512 elements, with a pitch ranging from $\lambda / 2$ to $3 \lambda / 2$ where $\lambda$ is the wavelength corresponding to the central frequency of the elements ${ }^{39}$. The pitch of the linear array used in this study was on the higher pitch range considering a central frequency between $5 \mathrm{MHz}$ and 7MHz.A smaller pitch would reduce the spatial averaging on the elements at frequencies higher than the central one and improve the image quality.On the other hand, linear arrays with central 
510 frequenciesranging from $30 \mathrm{MHz}$ to $65 \mathrm{MHz}{ }^{50-54}$ have been developed recently, and some are already

511 available commercially. Currently, to our knowledge, only linear and annular ${ }^{55,56}$ arrays have been

512 developed for such high frequencies. Array geometries where the elements are arranged on a concave

513 curved line or surface are a technical challenge at such frequencies. The high-frequency linear arrays

514 developed so far are moreovercylindrically focused to enhance their sensitivity, and tolimit spatial

515 averaging on thedetector surfacewithin the focal region. The novel detection geometry proposed here is

516 therefore adapted for these arrays,provided that the radial distance to the axis of rotation and the

517 translation steps are adapted to the array characteristics and the sample under study. Such a high

518 frequency system is expected to lead tovolumetricimages at mesoscopic scale of better quality than the

519 optoacoustic methods proposed so far, and will be investigated in the near future.Alternatively, a multi-

520 sector scanning scheme, as developed for two-dimensionnal imaging in $\operatorname{Ref}^{57}$, could be investigated with

521 an array. Suchscanning scheme resembles the fan beam scanning method for the third generation of 522 computed tomography $\mathrm{x}$-ray imaging ${ }^{40}$.

523

524

525

526

527

\section{V. CONCLUSION}

529

530

531

532 volume of the region of interest. interest.

To go beyond the frequencies available for arrays, spherically focused transducers could be used in similar detection geometry by translating the sensor along the elevation direction as well, or by scanning the tangent planes of a sphere centered on the sample. As for the array, the focal zone (volume of high sensitivity and bandwidth) shall be translated and rotated in order toefficiently sample in the entire

An original detection geometry for optoacoustic tomography, leading tohigh-resolutionvolumetric imaging,was presented in this work. The proposed geometry is based on the combination of a translaterotate scanning arrangement and the use of a conventional ultrasound linear array. This combination provides a large solid angular detection aperture and versatility in terms of dimensions of the region-of-

The implementation of the geometry with a conventional medical ultrasound linear array, designed to perform ultrasonography at 5.0/7.0 MHz, showed that whole-body volumetric optoacoustic images of mice could be performed, with imaged features correlating well to the mouse anatomy. The proposed geometry is therefore relevant for optoacoustic imaging of biological tissue. 
With the possibility to use high frequency detector arrays, the method presented herein is expected to be of great interest for volumetric imaging of optical absorption contrast at mesoscopic scale.The additional use of a multispectralapproach could moreover provide useful information on the three-dimensional location of specific and targeted chromophores in the anatomy.

542

\section{ACKNOWLEDGEMENTS}

The authors thank Dr Jing Shi from iThera Medicaland Dr Luis Xose Dean Benfor fruitful discussions.We would also like to thankSarah Glasl and Florian Jurgeleitfor the laboratory mouse handling. Vasilis Ntziachristos acknowledges funding from an ERC Advanced Investigator Award and BMBF.

\section{REFERENCES}

1. V. Ntziachristos, "Going deeper than microscopy: the optical imaging frontier in biology," Nat Meth 7, 603-614 (2010).

2. M. Xu and L. V. Wang, "Photoacoustic imaging in biomedicine," Review of Scientific Instruments 77, 041101-041122 (2006).

3. X. L. Deán-Ben, R. Daniel and N. Vasilis, "The effects of acoustic attenuation in optoacoustic signals," Physics in Medicine and Biology 56, 6129 (2011).

4. M. J. Niedre, R. H. de Kleine, E. Aikawa, D. G. Kirsch, R. Weissleder and V. Ntziachristos, "Early photon tomography allows fluorescence detection of lung carcinomas and disease progression in mice in vivo," Proceedings of the National Academy of Sciences 105, 1912619131 (2008).

5. M. Xu and L. V. Wang, "Analytic explanation of spatial resolution related to bandwidth and detector aperture size in thermoacoustic or photoacoustic reconstruction," Physical Review E 67, 056605 (2003).

6. H.-P. Brecht, R. Su, M. Fronheiser, S. A. Ermilov, A. Conjusteau and A. A. Oraevsky, "Wholebody three-dimensional optoacoustic tomography system for small animals," Journal of biomedical optics 14, 064007-064008 (2009).

7. R. B. Lam, R. A. Kruger, D. R. Reinecke, S. P. DelRio, M. M. Thornton, P. A. Picot and T. G. Morgan, "Dynamic optical angiography of mouse anatomy using radial projections," Photons Plus Ultrasound: Imaging and Sensing 2010, San Francisco, California, USA, 7564, 756405756407, (2010).

8. X. Wang, D. L. Chamberland, P. L. Carson, J. B. Fowlkes, R. O. Bude, D. A. Jamadar and B. J. Roessler, "Imaging of joints with laser-based photoacoustic tomography: An animal study," Medical Physics 33, 2691-2697 (2006).

9. R. A. Kruger, W. L. Kiser, D. R. Reinecke, G. A. Kruger and K. D. Miller, "Thermoacoustic Molecular Imaging of Small Animals " Molecular Imaging 2, 113-123 (2003).

10. D. Razansky, A. Buehler and V. Ntziachristos, "Volumetric real-time multispectral optoacoustic tomography of biomarkers," Nature protocols 6, 1121-1129 (2011). 
612

613

614

615

616

617

618

619

620

621

622

623

624

11. E. Z. Zhang, J. G. Laufer, R. B. Pedley and P. C. Beard, "In vivo high-resolution 3D photoacoustic imaging of superficial vascular anatomy," Physics in Medicine and Biology 54, 1035 (2009).

12. J. Laufer, E. Zhang, G. Raivich and P. Beard, "Three-dimensional noninvasive imaging of the vasculature in the mouse brain using a high resolution photoacoustic scanner," Appl. Opt. 48, D299-D306 (2009).

13. R. A. Kruger, R. B. Lam, D. R. Reinecke, S. P. Del Rio and R. P. Doyle, "Photoacoustic angiography of the breast," Medical Physics 37, 6096-6100 (2010).

14. J. Xia, Z. Guo, K. Maslov, A. Aguirre, Q. Zhu, C. Percival and L. V. Wang, "Three-dimensional photoacoustic tomography based on the focal-line concept," Journal of biomedical optics 16, 090505-090503 (2011).

15. L. Song, K. Maslov, R. Bitton, K. K. Shung and L. V. Wang, "Fast 3-D dark-field reflectionmode photoacoustic microscopy in vivo with a $30-\mathrm{MHz}$ ultrasound linear array," Journal of biomedical optics 13, 054028-054025 (2008).

16. M. P. Fronheiser, S. A. Ermilov, H.-P. Brecht, A. Conjusteau, R. Su, K. Mehta and A. A. Oraevsky, "Real-time optoacoustic monitoring and three-dimensional mapping of a human arm vasculature," Journal of biomedical optics 15, 021305-021307 (2010).

17. L. Song, C. Kim, K. Maslov, K. K. Shung and L. V. Wang, "High-speed dynamic 3D photoacoustic imaging of sentinel lymph node in a murine model using an ultrasound array," Medical Physics 36, 3724-3729 (2009).

18. A. Buehler, E. Herzog, A. Ale, B. D. Smith, V. Ntziachristos and D. Razansky, "High resolution tumor targeting in living mice by means of multispectral optoacoustic tomography," EJNMMI research 2, 14 (2012).

19. P. V. Chitnis, H.-P. Brecht, R. Su and A. A. Oraevsky, "Feasibility of optoacoustic visualization of high-intensity focused ultrasound-induced thermal lesions in live tissue," Journal of biomedical optics 15, 021313-021315 (2010).

20. J. Laufer, P. Johnson, E. Zhang, B. Treeby, B. Cox, B. Pedley and P. Beard, "In vivo preclinical photoacoustic imaging of tumor vasculature development and therapy," Journal of biomedical optics 17, 056016-056018 (2012).

21. P. Beard, "Biomedical photoacoustic imaging," Interface Focus 1, 602-631 (2011).

22. R. A. Kruger, P. Liu, Y. R. Fang and C. R. Appledorn, "Photoacoustic ultrasound (PAUS)--Reconstruction tomography," Medical Physics 22, 1605-1609 (1995).

23. R. Ma, A. Taruttis, V. Ntziachristos and D. Razansky, "Multispectral optoacoustic tomography (MSOT) scanner for whole-body small animal imaging," Opt. Express 17, 21414-21426 (2009).

24. D. Razansky, M. Distel, C. Vinegoni, R. Ma, N. Perrimon, R. W. Koster and V. Ntziachristos, "Multispectral opto-acoustic tomography of deep-seated fluorescent proteins in vivo," Nat Photon 3, 412-417 (2009).

25. S. Gratt, K. Passler, R. Nuster and G. Paltauf, "Photoacoustic section imaging with an integrating cylindrical detector," Biomed. Opt. Express 2, 2973-2981 (2011).

26. A. Buehler, E. Herzog, D. Razansky and V. Ntziachristos, "Video rate optoacoustic tomography of mouse kidney perfusion," Opt. Lett. 35, 2475-2477 (2010).

27. J. Gamelin, A. Aguirre, A. Maurudis, F. Huang, D. Castillo, L. V. Wang and Q. Zhu, "Curved array photoacoustic tomographic system for small animal imaging," Journal of biomedical optics 13, 024007-024010 (2008).

28. R. A. Kruger, J. W. L. Kiser, D. R. Reinecke and G. A. Kruger, "Thermoacoustic computed tomography using a conventional linear transducer array," Medical Physics 30, 856-860 (2003).

29. E. Zhang, J. Laufer and P. Beard, "Backward-mode multiwavelength photoacoustic scanner using a planar Fabry-Perot polymer film ultrasound sensor for high-resolution three-dimensional imaging of biological tissues," Appl. Opt. 47, 561-577 (2008). 
661

662

663

664

665

666

667

668

669

670

671

672

30. R. J. Zemp, L. Song, R. Bitton, K. K. Shung and L. V. Wang, "Realtime photoacoustic microscopy in vivo with a 30-MHz ultrasound array transducer," Opt. Express 16, 7915-7928 (2008).

31. Y. Xu, L. V. Wang, G. Ambartsoumian and P. Kuchment, "Reconstructions in limited-view thermoacoustic tomography," Medical Physics 31, 724-733 (2004).

32. A. Buehler, A. Rosenthal, T. Jetzfellner, A. Dima, D. Razansky and V. Ntziachristos, "Modelbased optoacoustic inversions with incomplete projection data," Med Phys 38, 1694-1704 (2011).

33. A. Rosenthal, V. Ntziachristos and D. Razansky, "Model-based optoacoustic inversion with arbitrary-shape detectors," Med Phys 38, 4285-4295 (2011).

34. G. J. Diebold and T. Sun, "Properties of Photoacoustic Waves in One, Two, and Three Dimensions," Acta Acustica united with Acustica 80, 339-351 (1994).

35. J. J. Niederhauser, M. Jaeger, R. Lemor, P. Weber and M. Frenz, "Combined ultrasound and optoacoustic system for real-time high-contrast vascular imaging in vivo," Medical Imaging, IEEE Transactions on 24, 436-440 (2005).

36. D. W. Yang, D. Xing, S. H. Yang and L. Z. Xiang, "Fast full-view photoacoustic imaging by combined scanning with a linear transducer array," Opt. Express 15, 15566-15575 (2007).

37. N. Felix, L. Ratsimandresy and L. Dufait, "High bandwidth, high density arrays for advanced ultrasound imaging," Ultrasonics Symposium, 2001 IEEE, 2, 1123-1126, (2001).

38. T. D. Khokhlova, I. M. Pelivanov and A. A. Karabutov, "Optoacoustic tomography utilizing focused transducers: The resolution study," Applied Physics Letters 92, 024105-024103 (2008).

39. K. K. Shung and M. Zippuro, "Ultrasonic transducers and arrays," Engineering in Medicine and Biology Magazine, IEEE 15, 20-30 (1996).

40. L. Goldman, "Principles of CT and CT technology," Journal of nuclear medicine technology $\mathbf{3 5}$, 115-128 (2007).

41. V. Ntziachristos and D. Razansky, "Molecular Imaging by Means of Multispectral Optoacoustic Tomography (MSOT)," Chemical Reviews 110, 2783-2794 (2010).

42. A. Rosenthal, V. Ntziachristos and D. Razansky, "Optoacoustic methods for frequency calibration of ultrasonic sensors," Ultrasonics, Ferroelectrics and Frequency Control, IEEE Transactions on 58, 316-326 (2011).

43. M. Xu and L. V. Wang, "Universal back-projection algorithm for photoacoustic computed tomography," Physical Review E 71, 016706 (2005).

44. B. A. J. Angelsen, H. Torp, S. Holm, K. Kristoffersen and T. A. Whittingham, "Which transducer array is best?," European Journal of Ultrasound 2, 151-164 (1995).

45. L. Changhui and V. W. Lihong, "Photoacoustic tomography and sensing in biomedicine," Physics in Medicine and Biology 54, R59 (2009).

46. M. I. Khan and G. J. Diebold, "The photoacoustic effect generated by an isotropic solid sphere," Ultrasonics 33, 265-269 (1995).

47. M. I. Khan and G. J. Diebold, "The photoacoustic effect generated by laser irradiation of an isotropic solid cylinder," Ultrasonics 34, 19-24 (1996).

48. T. Iwaki, H. Yamashita and T. Hayakawa, A Color Atlas of Sectional Anatomy of the Mouse. (アドスリー, 2001).

49. T. L. Szabo, Diagnostic ultrasound imaging : inside out. (Elsevier Academic Press, Amsterdam ; Boston, 2004).

50. L. Changgeng, Z. Qifa, F. T. Djuth and K. K. Shung, "High-frequency $(>50 \mathrm{MHz})$ medical ultrasound linear arrays fabricated from micromachined bulk PZT materials," Ultrasonics, Ferroelectrics and Frequency Control, IEEE Transactions on 59, 315-318 (2012).

51. J. A. Brown, F. S. Foster, A. Needles, E. Cherin and G. R. Lockwood, "Fabrication and Performance of a 40-MHz Linear Array Based on a 1-3 Composite with Geometric Elevation 
Focusing," Ultrasonics, Ferroelectrics and Frequency Control, IEEE Transactions on 54, 18881894 (2007).

52. J. M. Cannata, J. A. Williams, Z. Qifa, T. A. Ritter and K. K. Shung, "Development of a 35-MHz piezo-composite ultrasound array for medical imaging," Ultrasonics, Ferroelectrics and Frequency Control, IEEE Transactions on 53, 224-236 (2006).

53. F. S. Foster, J. Mehi, M. Lukacs, D. Hirson, C. White, C. Chaggares and A. Needles, "A New 15$50 \mathrm{MHz}$ Array-Based Micro-Ultrasound Scanner for Preclinical Imaging," Ultrasound in Medicine \&amp; Biology 35, 1700-1708 (2009).

54. S. Michau, P. Mauchamp and R. Dufait, "Piezocomposite $30 \mathrm{MHz}$ linear array for medical imaging: design challenges and performances evaluation of a 128 elements array," Ultrasonics Symposium, 2004 IEEE, 2, 898-901, (2004).

55. J. A. Brown, C. E. M. Demore and G. R. Lockwood, "Design and fabrication of annular arrays for high-frequency ultrasound," Ultrasonics, Ferroelectrics and Frequency Control, IEEE Transactions on 51, 1010-1017 (2004).

56. E. J. Gottlieb, J. M. Cannata, H. Chang-Hong and K. K. Shung, "Development of a highfrequency $(>50 \mathrm{MHz})$ copolymer annular-array, ultrasound transducer," Ultrasonics, Ferroelectrics and Frequency Control, IEEE Transactions on 53, 1037-1045 (2006).

57. M. Xu, G. Ku and L. V. Wang, "Microwave-induced thermoacoustic tomography using multisector scanning," Medical Physics 28, 1958-1963 (2001). 